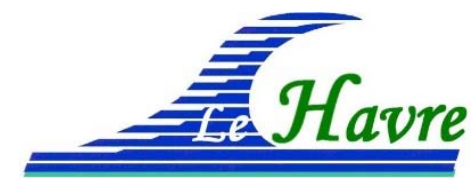

XVI ${ }^{\text {èmes }}$ Journées Nationales Génie Côtier - Génie Civil
Le Havre, 2020

DOI:10.5150/jngcgc.2020.007 (C) Editions Paralia CFL

disponible en ligne - http://www.paralia.fr - available online

\title{
Étude de l'effet de la non-linéarité des vagues sur le setup suivant une approche semi-analytique à phase moyennée
}

\author{
Thomas GUÉRIN ${ }^{1}$
}

\author{
1. SAS Benoit Waeles - Consultant Génie Côtier, 53 rue du Commandant Groix, \\ 29200 Brest, France. \\ thomas.guerin@bw-cgc.fr
}

\section{Résumé :}

Le setup, i.e. l'augmentation du niveau moyen à la côte due au déferlement des vagues, est un processus observé depuis plus de 50 ans et son importance en dynamique côtière, en particulier lors d'évènements extrêmes, est bien identifiée. La modélisation du setup au sein d'approches à phase moyennée est, du fait de la relative simplicité de son implémentation dans les modèles, souvent basée sur le concept des tensions de radiation en considérant implicitement les vagues incidentes comme des ondes sinusoïdales (i.e. vagues linéaires suivant la théorie d'Airy). Bien que cette approximation devienne clairement de plus en plus grossière à mesure que (depuis le large) l'on se rapproche du point de déferlement et de la zone de surf, relativement peu d'études ont déjà considéré l'incidence de cette approximation lors de la modélisation du setup suivant une approche à phase moyennée. Dans le but d'éclaircir ce point, la présente étude propose une comparaison entre l'approche classique linéaire précédemment décrite et une approche basée sur la fonction de courant (méthode initialement introduite par DEAN (1965)), cette dernière permettant de prendre en compte le caractère non-linéaire des vagues au sein du calcul des tensions de radiation. Une approche semi-analytique (1D cross-shore) est employée dans ce travail, tandis que les données de laboratoire (canal à houle) issues du projet GLOBEX (RUESSINK et al., 2013) sont exploitées afin de quantifier la pertinence des deux approches théoriques vis-à-vis de la prédiction du setup.

\section{Mots-clés :}

Vagues, Setup, Non-linéarité, Tensions de radiation, Modèles à phase moyennée, GLOBEX.

\section{Introduction}

Le setup, i.e. l'augmentation du niveau moyen à la côte due au déferlement des vagues, est un processus bien connu dans le domaine de l'hydrodynamique côtière, les premières observations et mesures remontant notamment aux travaux de SAVAGE (1957) et SAVILLE (1961). En particulier, lors d'évènements tempétueux, l'importante contribution du setup aux niveaux d'eau extrêmes à la côte est bien identifiée (e.g. NICOLAE LERMA et al., 2017 ; GUÉRIN et al., 2018 ; DODET et al., 2019). L'emploi de modèles de vague à phase moyennée en dynamique côtière étant assez répandu 


\section{Thème 1 - Hydrodynamique côtière}

(principalement du fait de leur pertinence en termes de temps de calcul vis-à-vis des échelles de temps et d'espace considérées), il est intéressant de noter que le calcul du setup au sein de ces modèles est souvent basé sur le concept des tensions de radiation (LONGUET-HIGGINS \& STEWART, 1964) associé à une représentation linéaire sinusoïdale des vagues (théorie d'Airy), malgré le fait que cette approximation apparaisse de plus en plus inappropriée à mesure que les vagues incidentes atteignent la zone de déferlement.

Bien que quelques travaux aient déjà considéré la prise en compte du caractère nonlinéaire des vagues lors du calcul des tensions de radiation (e.g. JAMES, 1974 ; WANG et al., 2008 ; SHABANI et al., 2011), l'effet de cette non-linéarité sur le calcul du setup n'a, à notre connaissance, pas spécifiquement été traité. La présente étude propose donc une comparaison entre l'utilisation de l'approche classique linéaire et une approche nonlinéaire basée sur le travail de DEAN (1965) lors du calcul du setup, suivant un modèle semi-analytique 1D cross-shore. Afin de juger de la pertinence des deux approches théoriques, les données de laboratoire issues du projet GLOBEX (RUESSINK et al., 2013) sont exploitées.

\section{Matériel et méthode}

\subsection{Modèle théorique}

Le modèle 1D cross-shore utilisé dans notre cas repose sur l'équation d'équilibre de la quantité de mouvement associée à la propagation des vagues suivant l'axe cross-shore :

$\frac{\partial S_{x x}}{\partial x}+\tau_{b}=-\rho g h \frac{\partial \bar{\eta}}{\partial x}$

où $x$ est la coordonnée suivant l'axe cross-shore, $S_{x x}$ l'excès de flux de quantité de mouvement dû à la présence des vagues (seule composante non nulle du tenseur des contraintes radiatives associé), $\tau b$ le frottement au fond (calculé dans notre cas suivant la méthode de MALARKEY \& DAVIS (2012)), $\rho$ la densité de l'eau, $g$ l'accélération gravitationnelle, $\bar{\eta}$ l'élévation moyenne de la surface libre, et $h$ la hauteur d'eau totale (i.e. somme de $\bar{\eta}$ et de la hauteur d'eau du fluide au repos $d$ ).

De manière analogue aux travaux de APOTSOS et al. (2007) par exemple, le terme $S_{x x}$ inclue la contribution du rouleau de déferlement qui dans notre cas est estimée suivant la méthode de LIPPMANN et al. (1996). Nous avons donc au final $S_{x x}=S_{x x, w}+S_{x x, r}$ où :

$S_{x x, w}=\overline{\int_{-d}^{\eta}\left(p+\rho u^{2}\right) d z}-\int_{-d}^{0} p_{0} d z$

et

$S_{x x, r}=\frac{1}{4} \rho c f \frac{H_{b}^{3}}{h \tan \alpha}$

avec $p$ la pression au sein du fluide ( $p_{0}$ la pression hydrostatique), $u$ la composante horizontale de la vitesse orbitale des vagues, $z$ la coordonnée verticale, $c$ la vitesse de phase des vagues, $f$ leur fréquence, $H_{b}$ la hauteur des vagues une fois déferlées (i.e. $H_{b}=0$ pour les vagues non déferlées), et $\alpha$ l'angle du front des vagues déferlées. 


\section{XVIèmes Journées Nationales Génie Côtier - Génie Civil \\ Le Havre 2020}

L'approche linéaire consiste donc à calculer $S_{x x}$ en considérant les variables caractérisant le mouvement des vagues (i.e. $p, u, \eta$ et $c$ ) telles qu'issues de la théorie d'Airy, tandis que l'approche non-linéaire choisie dans ce travail considère l'expression de ces variables obtenue au moyen de la méthode initialement proposée par DEAN (1965) et reposant sur l'utilisation de la fonction de courant (méthode appelée plus précisément stream function representation method ou Fourier approximation method). Cette méthode ayant été améliorée à plusieurs reprises depuis son introduction par DEAN (1965), la version proposée par FENTON (1999) est ici utilisée pour le calcul des variables précédemment citées.

\subsection{Données GLOBEX}

Le projet Gently sLOping Beach EXperiments (GLOBEX) s'est déroulé à Deltares (Delft, Pays-Bas) en 2012, où différentes conditions de vagues ont été propagées au sein d'un canal à houle de $100 \mathrm{~m}$ de long caractérisé sur les premiers $15 \%$ offshore d'un fond plat définissant une hauteur d'eau de $0.85 \mathrm{~m}$, suivi pour les $85 \%$ restants d'une pente de plage uniforme de 1/80 jusqu'à la limite onshore. Ces expériences sont décrites en détails par RUESSINK et al. (2013). Dans le présent travail, les six principales séries d'expériences ont été considérées : les conditions bichromatiques B1, B2 et B3, ainsi que les conditions aléatoires A1, A2 et A3 (voir tableau 1).

Tableau 1. Conditions de vagues GLOBEX : amplitude (a), fréquence (f), période pic (Tp), période de groupe (Tg), et facteur d'amplification du pic d'énergie ( $\gamma_{\text {JONSWAP). }}$

\begin{tabular}{cccccccc} 
Série & $\boldsymbol{a}_{\mathbf{1}}(\mathbf{m})$ & $\boldsymbol{a}_{\mathbf{2}}(\mathbf{m})$ & $\boldsymbol{f}_{\mathbf{1}}(\mathbf{H z})$ & $\boldsymbol{f}_{\mathbf{2}}(\mathbf{H z})$ & $\boldsymbol{T}_{\boldsymbol{p}}(\mathbf{s})$ & $\boldsymbol{T}_{\boldsymbol{g}}(\mathbf{s})$ & $\gamma_{\text {JONSWAP }}$ \\
\hline B1 & 0.09 & 0.01 & 0.40 & 0.467 & 2.308 & 15.0 & - \\
B2 & 0.09 & 0.01 & 0.42 & 0.462 & 2.268 & 23.81 & - \\
B3 & 0.07 & 0.03 & 0.42 & 0.462 & 2.268 & 23.81 & - \\
A1 & 0.05 & - & - & - & 1.60 & - & 3.3 \\
A2 & 0.10 & - & - & - & 2.25 & - & 3.3 \\
A3 & 0.05 & - & - & - & 2.25 & - & 20 \\
\hline
\end{tabular}

\section{Résultats}

Les comparaisons entre théorie et mesures présentées ci-après concernent les 5 premières minutes de chaque expérience, tandis que seuls les points de l'axe $x$ où, à la fois l'élévation de surface et la vitesse orbitale ont été mesurées, sont considérés.

\section{1 Élévation de la surface libre}

Un exemple de comparaison entre élévation de la surface libre mesurée et prédite suivant les deux approches théoriques est présenté en figure 1, tandis que l'erreur quadratique moyenne normalisée (EQMN) associée aux prédictions théoriques et calculée aux différents points de mesure cross-shore est présentée en figure 2. Une nette diminution de 


\section{Thème 1 - Hydrodynamique côtière}

l'EQMN sur l'ensemble des points de mesure est constatée lorsque l'approche nonlinéaire est considérée, synonyme d'une meilleure représentation de l'élévation de la surface libre. La performance des deux approches théoriques pour l'ensemble des conditions expérimentales est synthétisée en tableau 2, où l'on constate une diminution systématique de l'EQMN lorsque l'on passe de la prédiction linéaire à non-linéaire.

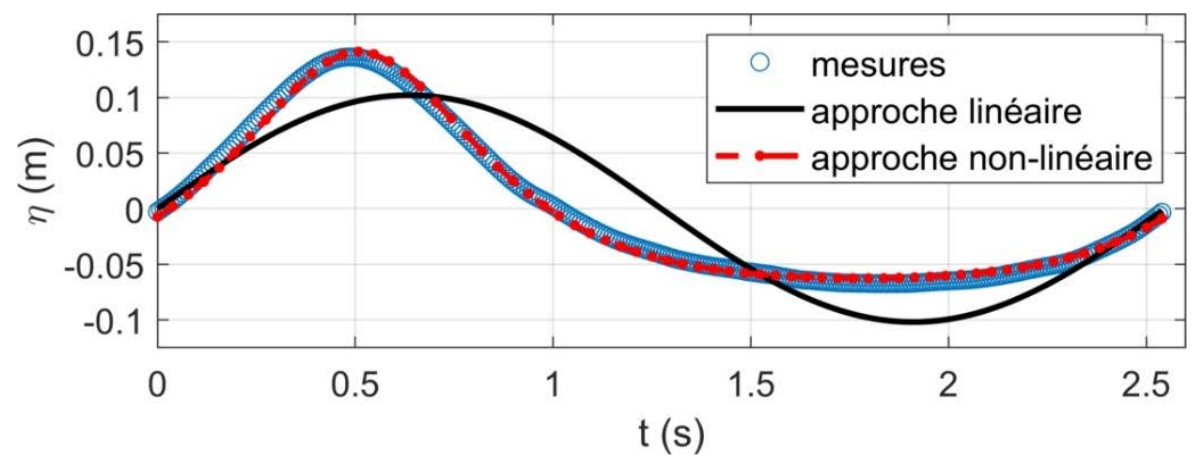

Figure 1. Exemple de comparaison entre un cycle orbital d'élévation de la surface libre

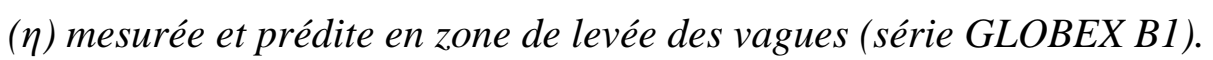

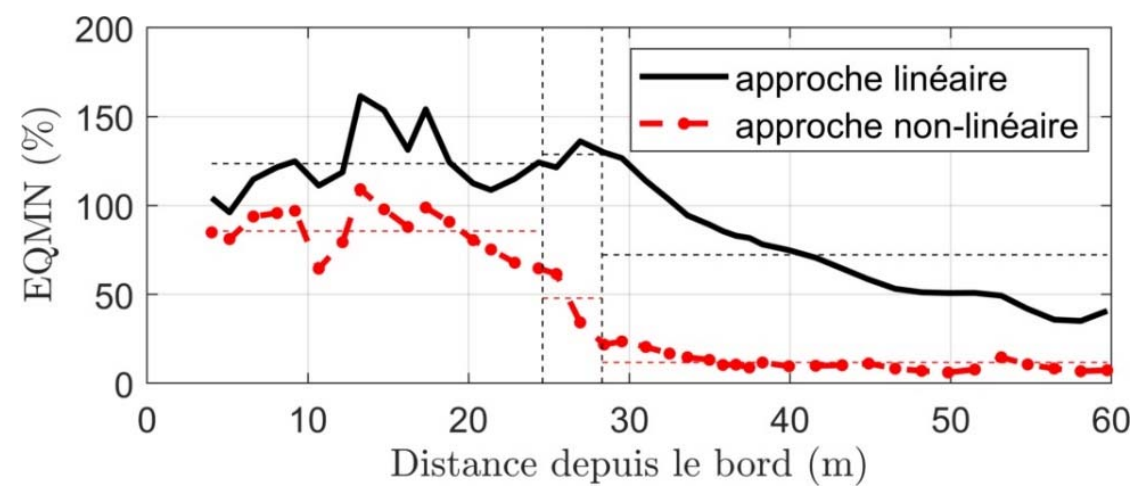

Figure 2. Exemple de profil cross-shore d'erreur quadratique moyenne normalisée (EQMN) pour $\eta$ (série GLOBEX B1). Les traits pointillés verticaux délimitent la zone offshore, de déferlement, et de surf ; les pointillés horizontaux correspondent aux valeurs moyennes d'EQMN par zone et pour chaque approche. 


\section{XVIèmes Journées Nationales Génie Côtier - Génie Civil \\ Le Havre 2020}

Tableau 2. Synthèse de la performance (EQMN) des approches linéaires (L) et nonlinéaires (NL) pour la prédiction de $\eta$, par zone et pour les 6 séries GLOBEX.

\begin{tabular}{ccccccc}
\hline \multirow{2}{*}{ Série } & \multicolumn{2}{c}{ Zone offshore } & \multicolumn{2}{c}{ Zone de déferlement } & \multicolumn{2}{c}{ Zone de surf } \\
\cline { 2 - 7 } & $\boldsymbol{L}$ & $\boldsymbol{N L}$ & $\boldsymbol{L}$ & $\boldsymbol{N L}$ & $\boldsymbol{L}$ & $\boldsymbol{N L}$ \\
\hline B1 & $72.3 \%$ & $11.7 \%$ & $128.8 \%$ & $47.9 \%$ & $123.6 \%$ & $85.6 \%$ \\
B2 & $62.3 \%$ & $9.4 \%$ & $121.4 \%$ & $41.7 \%$ & $115.0 \%$ & $87.4 \%$ \\
B3 & $53.1 \%$ & $14.6 \%$ & $112.6 \%$ & $60.7 \%$ & $134.5 \%$ & $99.0 \%$ \\
A1 & $48.0 \%$ & $42.9 \%$ & $96.8 \%$ & $60.1 \%$ & $129.5 \%$ & $87.2 \%$ \\
A2 & $55.1 \%$ & $47.5 \%$ & $81.7 \%$ & $56.6 \%$ & $126.5 \%$ & $89.5 \%$ \\
A3 & $45.1 \%$ & $36.1 \%$ & - & - & $112.5 \%$ & $73.7 \%$ \\
\hline
\end{tabular}

\subsection{Composante horizontale de la vitesse orbitale}

De manière analogue à la variable d'élévation de la surface libre, la performance des deux approches théoriques quant à la prédiction de la composante horizontale de la vitesse orbitale est analysée (voir figures 3 et 4, et tableau 3). Une amélioration systématique de la prédiction de cette variable lorsque l'approche non-linéaire est considérée est également obtenue.

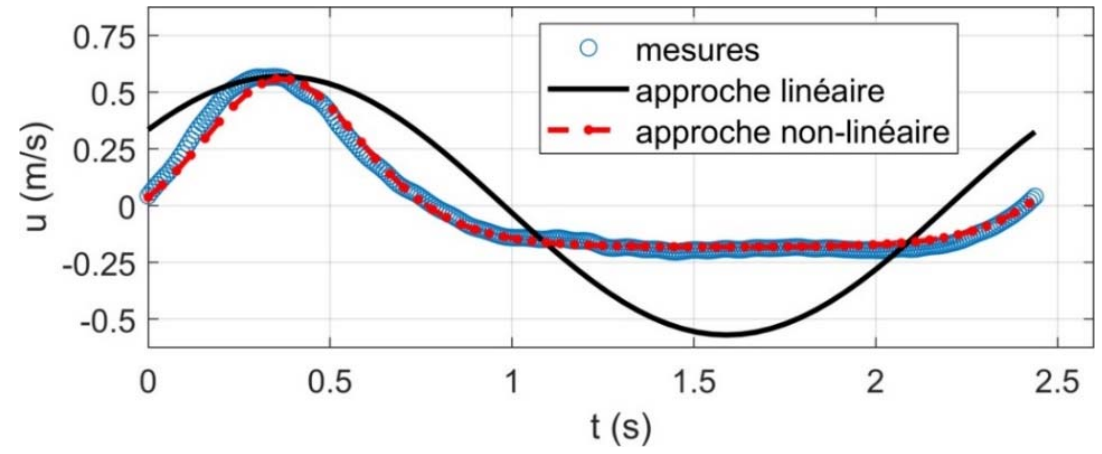

Figure 3. Exemple de comparaison entre un cycle orbital de vitesse horizontale (u) mesurée et prédite en zone de levée des vagues et près du fond (série GLOBEX B1).

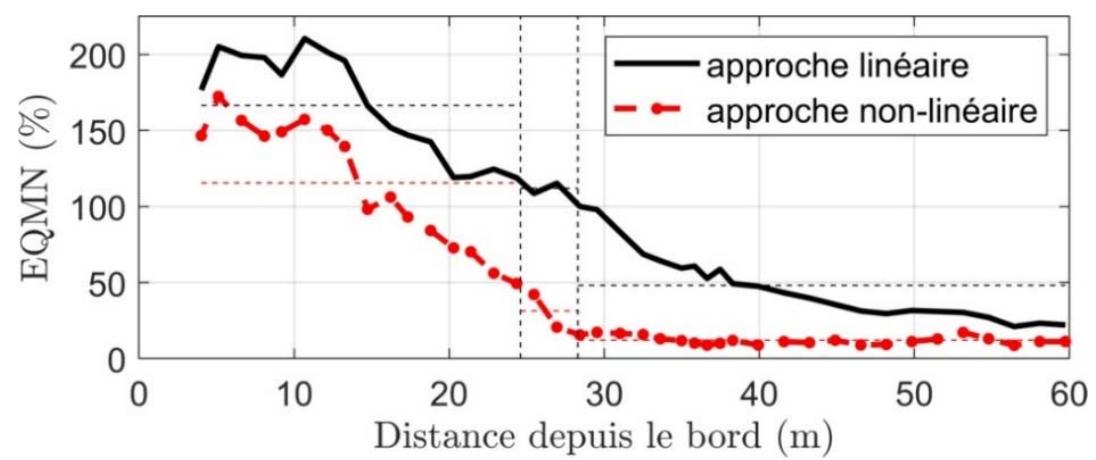

Figure 4. Exemple de profil cross-shore d'erreur quadratique moyenne normalisée (EQMN) pour u (série GLOBEX B1). 


\section{Thème 1 - Hydrodynamique côtière}

Tableau 3. Synthèse de la performance (EQMN) des approches linéaires (L) et nonlinéaires (NL) pour la prédiction de u, par zone et pour les 6 séries GLOBEX.

\begin{tabular}{ccccccc}
\hline \multirow{2}{*}{ Série } & \multicolumn{2}{c}{ Zone offshore } & \multicolumn{2}{c}{ Zone de déferlement } & \multicolumn{2}{c}{ Zone de surf } \\
\cline { 2 - 7 } & $\boldsymbol{L}$ & $\boldsymbol{N L}$ & $\boldsymbol{L}$ & $\boldsymbol{N L}$ & $\boldsymbol{L}$ & $\boldsymbol{N L}$ \\
\hline B1 & $48.1 \%$ & $12.0 \%$ & $111.9 \%$ & $31.4 \%$ & $166.4 \%$ & $115.5 \%$ \\
B2 & $39.7 \%$ & $11.2 \%$ & $104.5 \%$ & $31.7 \%$ & $169.2 \%$ & $127.3 \%$ \\
B3 & $37.3 \%$ & $16.6 \%$ & $103.8 \%$ & $50.5 \%$ & $177.9 \%$ & $130.6 \%$ \\
A1 & $47.8 \%$ & $42.2 \%$ & $89.8 \%$ & $56.2 \%$ & $136.3 \%$ & $90.6 \%$ \\
A2 & $52.5 \%$ & $48.1 \%$ & $76.4 \%$ & $53.4 \%$ & $145.6 \%$ & $98.9 \%$ \\
A3 & $44.0 \%$ & $39.4 \%$ & - & - & $116.2 \%$ & $75.7 \%$ \\
\hline
\end{tabular}

\subsection{Elévation moyenne de la surface libre}

Une fois les termes $S_{x x, w}$ et $S_{x x, r}$ calculés de manière semi-analytique suivant les deux approches théoriques (i.e. considérant les variables $p(x, z, t), u(x, z, t), \eta(x, t)$ et $c(x)$ fournies séparément par ces deux approches dans les équations (2) et (3), et en discrétisant suivant $z$ et $t$ pour le calcul de $S_{x x, w}$ ), l'équation (1) est résolue au moyen d'un schéma de type différences finies et centré en espace. La condition à la limite offshore est telle que $\bar{\eta}$ prédit est égale à $\bar{\eta}$ mesuré pour les deux premiers points de l'axe $x$ en partant de cette limite offshore. De plus, du fait qu'au sein de l'équation (1) la hauteur d'eau totale $h$ soit dépendante de $\bar{\eta}$, une boucle itérative sur $h$ est considérée pour s'assurer de la convergence de la solution (i.e. l'équation (1) est résolue de manière itérative en actualisant $h$ une fois $\bar{\eta}(x)$ obtenu; en général moins de 5 itérations suffisent pour converger). Le profil cross-shore d'élévation moyenne de la surface libre est ainsi obtenu pour les deux approches et comparé au profil mesuré (voir figure 5).

En se focalisant sur la zone de surf (i.e. zone de setup), et plus particulièrement en limite de la ligne d'eau (i.e. près du bord où le setup est maximal), on constate une amélioration significative de la prédiction du setup lorsque l'approche non-linéaire est considérée. Plus précisément, la surestimation systématique du setup obtenue avec l'approche linéaire, principalement due à la surestimation de l'amplitude de la vitesse orbitale horizontale (le terme $S_{x x, w}$ dépendant de $u^{2}$ ), se voit globalement diminuée d'un facteur 2 à 4 lorsque l'approche non-linéaire est considérée (voir tableau 4).

Il est également important de préciser que, dans le cas des expériences GLOBEX considérées dans la présente étude, la contribution du gradient de $S_{x x, w}$ au sein de la partie gauche de l'équation (1) (ou partie « forçage » de l'équation) apparait prépondérante en comparaison de la contribution du rouleau de déferlement et du frottement au fond (i.e. gradient de $S_{x x, r}$ et $\tau_{b}$; non montré). Autrement dit le profil du setup apparaît largement contraint par le profil de $S_{x x, w}$, et en (bien) moindre mesure par le frottement au fond ainsi que par le profil de $S_{x x, r}$. 

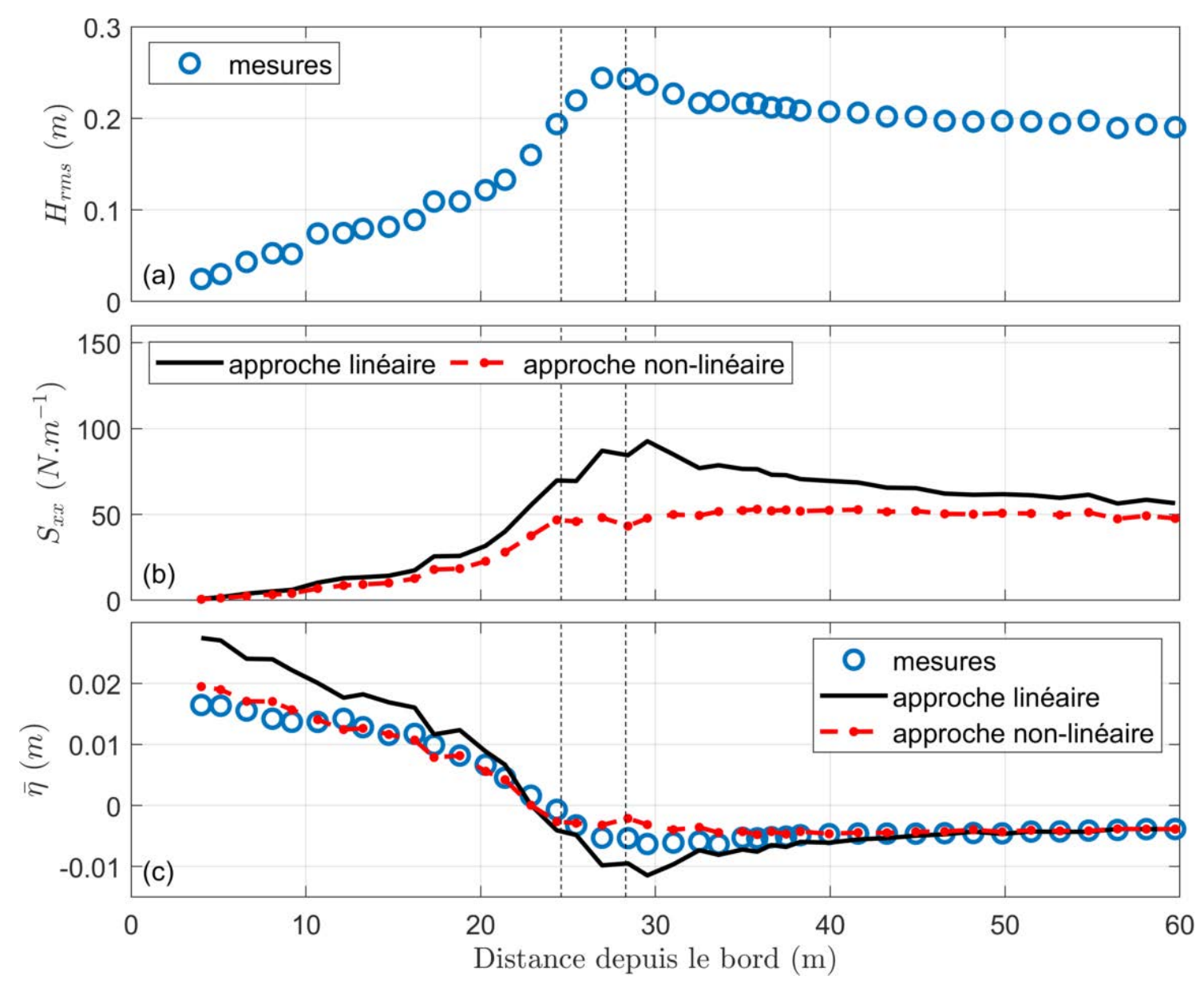

Figure 5. Exemple (série GLOBEX B1) de profil cross-shore obtenu pour : (a) Hrms mesuré, (b) $S_{x x}$ prédit, et (c) $\bar{\eta}$ mesuré et prédit.

Tableau 4. Synthèse de la performance (EQMN) des approches linéaires $(L)$ et nonlinéaires (NL) pour la prédiction du setup, en zone de surf et au bord, pour les 6 séries GLOBEX.

\begin{tabular}{ccccc}
\hline \multirow{2}{*}{ Série } & \multicolumn{2}{c}{ Zone de surf } & \multicolumn{2}{c}{ Au bord } \\
\cline { 2 - 5 } & $\boldsymbol{L}$ & $\boldsymbol{N L}$ & $\boldsymbol{L}$ & $\boldsymbol{N L}$ \\
\hline B1 & $59.1 \%$ & $15.8 \%$ & $65.9 \%$ & $17.4 \%$ \\
B2 & $71.9 \%$ & $23.4 \%$ & $66.7 \%$ & $19.8 \%$ \\
B3 & $76.1 \%$ & $27.5 \%$ & $80.5 \%$ & $30.7 \%$ \\
A1 & $80.0 \%$ & $33.2 \%$ & $82.6 \%$ & $36.1 \%$ \\
A2 & $109.1 \%$ & $53.7 \%$ & $98.8 \%$ & $46.6 \%$ \\
A3 & $146.9 \%$ & $82.5 \%$ & $85.5 \%$ & $34.5 \%$ \\
\hline
\end{tabular}




\section{Thème 1 - Hydrodynamique côtière}

\section{Conclusion}

De manière finalement assez attendue au vu de la différence de représentation des deux principales variables ( $\eta$ et $u$ ) caractérisant les vagues d'une approche théorique à l'autre (voir figures 1 et 3), la présente étude montre que la prise en compte du caractère nonlinéaire des vagues lors du calcul des tensions de radiation améliore significativement la prédiction du setup par rapport à l'approche basée sur une représentation linéaire sinusoïdale des vagues. En particulier, concernant les expériences GLOBEX tout du moins, la prise en compte de la non-linéarité des vagues entraîne in fine une diminution du setup prédit (via principalement une diminution du terme « radiatif » $S_{x x, w}$ ) par rapport au cas linéaire. Ce résultat est notamment en accord avec les travaux de WANG et al. (2008) et SHABANI et al. (2011), bien que l'effet de la non-linéarité des vagues sur le setup soit relativement plus important dans le présent travail.

À la suite de ce constat il est intéressant de noter que le calcul des tensions de radiation dans nombre de modèles spectraux de vagues (e.g. WWM, SWAN, WAVEWATCH III) est basé, de manière intrinsèque, sur une représentation linéaire sinusoïdale des vagues. En effet la formulation spectrale des tensions de radiation formulée en chapitre 5 de BATTJES (1974), celle-ci s'appuyant sur l'expression analytique proposée par LONGUET-HIGGINS \& STEWART (1964) dans le cas de vagues monochromatiques représentées comme des ondes sinusoïdales, y est communément employée. Ces modèles spectraux étant fréquemment utilisés au sein de la communauté océanographique côtière pour simuler les niveaux extrêmes atteints à la côte lors d'événements tempétueux, il apparait pertinent de se questionner quant à l'effet potentiel de la non-linéarité des vagues sur le setup (et sur les courants induits par les vagues) ainsi modélisé(s).

\section{Remerciements}

Sont vivement remerciées toutes les personnes ayant été impliquées dans le projet GLOBEX, soutenu par le European Community's Seventh Framework Program au travers du contrat n ${ }^{\circ} 261520$ de 1'Integrating Activity HYDRALAB IV. Anouk de Bakker et Gerben Ruessink sont en particulier remerciés pour la mise à disposition des données GLOBEX. La SAS Benoit Waeles - Consultant Génie Côtier est sincèrement remerciée pour le financement de la part d'activité de recherche de l'auteur.

\section{Références bibliographiques}

APOTSOS A., RAUBENHEIMER B., ELGAR S., GUZA R.T., SMITH J.A. (2007). Effects of wave rollers and bottom stress on wave setup. Journal of Geophysical Research: Oceans, 112(C2). https://doi.org/10.1029/2006JC003549

BATTJES J.A. (1974). Computation of set-up, longshore currents, run-up, and overtopping due to wind-generated waves. Rep. 74-2, Comm. On Hydraul., Department of Civil Engineering, Delft University of Technology, Delft, Netherlands. 


\section{XVİ̀mes Journées Nationales Génie Côtier - Génie Civil \\ Le Havre 2020}

DEAN R.G. (1965). Stream function representation of nonlinear ocean waves. Journal of Geophysical Research, Vol. 70 (18), pp 4561-4572. https://doi.org/10.1029/JZ070i018p04561

DODET G., MELET A., ARDHUIN F., BERTIN X., IDIER D., ALMAR R. (2019). The contribution of wind-generated waves to coastal sea-level changes. Surveys in Geophysics, Vol. 40(6), pp 1563-1601. https://doi.org/10.1007/s10712-019-09557-5

FENTON J. D. (1999). Numerical methods for nonlinear waves. In Advances in coastal and ocean engineering, pp 241-324. https://doi.org/10.1142/9789812797544 0005

GUÉRIN T., BERTIN X., COULOMBIER T., DE BAKKER A. (2018). Impacts of waveinduced circulation in the surf zone on wave setup. Ocean Modelling, Vol. 123, pp 86-97. https://doi.org/10.1016/j.ocemod.2018.01.006

JAMES I.D. (1974). Non-linear waves in the nearshore region: shoaling and set-up. Estuarine and Coastal Marine Science, Vol. 2(3), pp 207-234. https://doi.org/10.1016/03023524(74)90013-9

LIPPMANN T.C., BROOKINS A.S., THORNTON E.B. (1996). Wave energy transformation on natural profiles. Coastal Engineering, Vol. 27(1-2), pp 1-20. https://doi.org/10.1016/0378-3839(95)00036-4

LONGUET-HIGGINS M. S., STEWART R. W. (1964). Radiation stresses in water waves; a physical discussion, with applications. Deep-Sea Research, Vol. 11(4), pp 529562. https://doi.org/10.1016/0011-7471(64)90001-4

MALARKEY J., DAVIES A. G. (2012). A simple procedure for calculating the mean and maximum bed stress under wave and current conditions for rough turbulent flow based on method. Computers \& Geosciences, Vol. 43, pp 101-107. https://doi.org/10.1016/j.cageo.2012.02.020

NICOLAE LERMA A., PEDREROS R., ROBINET A., SENECHAL N. (2017). Simulating wave setup and runup during storm conditions on a complex barred beach. Coastal Engineering, 123, pp 29-41. https://doi.org/10.1016/j.coastaleng.2017.01.011

RUESSINK B. G., MICHALLET H., BONNETON P., MOUAZE D., LARA J., SILVA P.A., WELLENS P. (2013). Globex: wave dynamics on a gently sloping laboratory beach. Proceedings of Coastal Dynamics 2013, pp 1351-1362.

SAVAGE R. P. (1957). Model tests for hurricane protection project. Bulletin of the Beach Erosion Board, US Army Corps of Engineers, Washington, DC.

SAVILLE T. (1961). Experimental determination of wave set-up. In Proc. 2nd Tech. Conf. on Hurricanes, Miami Beach, FL, 1961. US Dept. of Commerce.

SHABANI B., NIELSEN P., BALDOCK T. E. (2011). Wave setup: A Non-linear approach. Twenty-first International Offshore and Polar Engineering Conference, 19-24 June, Maui, Hawaii, USA, pp 853-860.

WANG B., CHADWICK A. J., OTTA A. K. (2008). Derivation and application of new equations for radiation stress and volume flux. Coastal engineering, Vol. 55(4), pp 302-318. https://doi.org/10.1016/j.coastaleng.2007.11.008 
Thème 1 - Hydrodynamique côtière 